\title{
Direct versus indirect processing changes the influence of color in natural scene categorization
}

\author{
SaChio Otsuka AND Jun KaWAguchi \\ Nagoya University, Nagoya, Japan
}

\begin{abstract}
We examined whether participants would use a negative priming (NP) paradigm to categorize color and grayscale images of natural scenes that were presented peripherally and were ignored. We focused on (1) attentional resources allocated to natural scenes and (2) direct versus indirect processing of them. We set up low and high attention-load conditions, based on the set size of the searched stimuli in the prime display (one and five). Participants were required to detect and categorize the target objects in natural scenes in a central visual search task, ignoring peripheral natural images in both the prime and probe displays. The results showed that, irrespective of attention load, NP was observed for color scenes but not for grayscale scenes. We did not observe any effect of color information in central visual search, where participants responded directly to natural scenes. These results indicate that, in a situation in which participants indirectly process natural scenes, color information is critical to object categorization, but when the scenes are processed directly, color information does not contribute to categorization.
\end{abstract}

Natural scenes are readily processed by the visual system. In their seminal article, Thorpe, Fize, and Marlot (1996) found that go versus no-go natural scene categorization was processed rapidly and accurately. Subsequent studies concerning natural scene categorization have reported similar results (e.g., Bacon-Macé, Macé, FabreThorpe, \& Thorpe, 2005; VanRullen \& Koch, 2003; VanRullen \& Thorpe, 2001a, 2001b). Li, VanRullen, Koch, and Perona (2002) reported that, as compared with letters and color patterns, rapid natural scene categorization required very little attention and that higher level representations could be accessed in the near absence of attention. Also, Evans and Treisman (2005) showed that, whereas object categorization in natural scenes did not require attention, identification did.

We recently examined whether natural scenes were categorized with minimal attentional resources by using a negative priming (NP) paradigm (Otsuka \& Kawaguchi, 2007; see also Lavie \& Fox, 2000). Generally, in an NP experiment, participants are required to respond to a target while ignoring a distractor in each trial. What is important is the relationship between the distractor in trial $n$ (i.e., the prime) and the target in trial $n+1$ (i.e., the probe). In the control (C) condition, no relation exists between the target and the distractor in the prime and probe trials. In the ignored repetition (IR) condition, the distractor ignored in the prime trial is presented as the target in the subsequent probe trial. In general, the reaction times (RTs) to the probe target in the IR condition are slower than those in the $\mathrm{C}$ condition. This delay is referred to as negative priming. The NP paradigm, used by Lavie and Fox, allowed us to measure the influence of the ignored images, which participants did not need to respond to. Our previous study compared natural scene categorization with letter discrimination and line drawing categorization. We set up low and high attention-load conditions, related to the size of the set of searched stimuli. In the low attention-load condition, one target and one distractor appeared in the prime display. In the high attention-load condition, one target was accompanied by four nontargets. On the prime display, all the distractors were incompatible with the response required to the current target, and all appeared randomly and equally either above or below the horizontal axis of the screen. In the $\mathrm{C}$ condition, the probe display contained a target and distractor that were different from those in the prime display. In the IR condition, a target on the probe display had the same identity as the distractor in the previous prime display. We predicted that, if people could categorize a target object in a natural scene with minimal attention, the NP effect should occur, irrespective of the attention load. The results for the RT data showed NP effects for natural scene categorization in both the low and high attentionload conditions, whereas neither letter discrimination nor line drawing categorization showed an NP effect in the high attention-load condition. This indicated that complex stimuli, such as natural scenes, were processed with minimal attentional resources and more readily than were simple stimuli, such as letters and line drawings. 
Most studies of natural scene categorization have used only colored images. However, some have investigated the effect of color information on natural scene recognition. Delorme, Richard, and Fabre-Thorpe (2000) examined the effect of color cues in natural scene categorization with humans and rhesus monkeys. They asked subjects to perform a categorization task (food vs. nonfood or animal vs. nonanimal). Their results showed that both colored and grayscale natural images produced similar RTs and accuracies, irrespective of the object categories. They argued that rapid natural scene categorization did not depend on the color information of natural images but on the feedforward processing of achromatic magnocellular information.

Rousselet, Joubert, and Fabre-Thorpe (2005) examined the color effect in natural scene categorization. They used a go versus no-go rapid categorization task with colored and grayscale natural scenes (sea, mountains, indoor and urban settings). The results showed that the colored and grayscale images were categorized with equally high accuracy $($ color $=95.1 \%$; grayscale $=94.7 \%)$. However, the RT data showed that people categorized the colored indoor images more rapidly than they did the grayscale indoor images and the colored sea images more rapidly than they did the grayscale sea images. Despite these exceptions, Rousselet et al. concluded that ultrarapid natural scene categorization was not based on the color information.

Fei-Fei, VanRullen, Koch, and Perona (2005) investigated the effect of color information in natural scenes using the procedure of Li et al. (2002). The results showed that the participants could perform rapid natural scene categorization with grayscale images in the dual- and single-task conditions. Their results indicated that people could categorize natural scenes without color information. They concluded that color information was not a critical factor in natural scene categorization in the near absence of attention.

In contrast, some studies reported the influence of color information in natural scene categorization (Castelhano \& Henderson, 2008; Goffaux et al., 2005; Oliva \& Schyns, 2000; Spence, Wong, Rusan, \& Rastegar, 2006). Goffaux et al. reported a different effect of color information in natural scene categorization. In their experiment, the color information of natural images was manipulated. In the normal condition, normally colored images were used as stimuli; in the grayscale condition, color information was removed; and in the abnormal condition, the images were obtained by swapping red and green pixels with one another and swapping blue and yellow pixels with one another. In a go versus no-go task, participants were asked to categorize natural images as "desert," "forest," "coastline," or "canyon." They measured RT, accuracy, and event-related potential (ERP). The behavioral data showed that mean RT in the normal condition was shorter than RT in the grayscale condition, which in turn was shorter than RT in the abnormal condition. Regarding accuracy, the mean percentage correct in the normal condition was higher than in the grayscale condition, which was higher than in the abnormal condition. Also, the averaged onset latencies of go versus no-go differences were consistently longer in the grayscale and abnormal conditions than in the normal condition.

Oliva and Schyns (2000) explored the influence of color information with color-diagnostic images and colornondiagnostic images. As with Goffaux et al. (2005), normal, grayscale, and abnormal conditions were compared as a function of color diagnosticity. Participants were asked to categorize natural images. Their results showed the color contribution to categorization with colordiagnostic images but not with color-nondiagnostic images. Also, the effect of color information was found with blurred-diagnostic images, as compared wtih blurrednondiagnostic images. ${ }^{1}$ Oliva and Schyns concluded that color is an important property of the relevant spatial layout that mediates express scene recognition.

More recently, in order to provide a more accurate measure of how quickly scene gist is acquired, Castelhano and Henderson (2008) examined the influence of color information with the contextual bias paradigm, in which the response bias is used as a measure of whether the gist of the scene is processed. They defined scene gist with the photographic scenes. All scenes were views of real-world environments that were scaled to a human size, corresponding to the types of views that people experience in their everyday lives. For these types of scenes, people have amassed knowledge of typical object contents, spatial layout, functions that can be performed in or around the scene, and other semantically related information. Thus, when enough information is extracted, the scene's basic-level category, the information regarding the expected scene content, and functions that can be performed within the scene are all activated. Castelhano and Henderson stated that the activation of this information is collectively referred to as the scene gist. In their experiments, participants are asked to indicate the presence of a target object whose consistency with the scene has been manipulated, but which is never present in the scenes. The logic of the contextual bias paradigm is that responses should be influenced by the activation of gist and that they should show a bias if the visual information acquired from a presentation of scenes is sufficient to activate scene gist. They asked participants to decide whether a target name presented in the display after the presentation of a natural image was in an image with multiple durations of natural images. The effect of color information was found in the blurred images but not in the normal images at longer durations $(80,100,250 \mathrm{msec})$. Castelhano and Henderson postulated that the color information helps to activate scene gist for blurred images but that it is not required to activate scene gist for normal images, so that normal images are sufficient to activate scene gist without color information.

Although the effect of color information depended on multiple factors of stimuli (e.g., normal vs. blurred and diagnostic vs. nondiagnostic), these results, on the whole, suggest that natural scene categorization is influenced by the color content of natural images. However, the role that color information plays in natural scene categorization remains unclear. In the present article, we address the relationship between the color influence of natural scene 
categorization and visual attention. Especially, it is possible that Fei-Fei et al.'s (2005) experiments permitted participants to use attentional resources because of direct processing of peripheral natural scenes and massive training. With regard to Fei-Fei et al.'s results, we can think of two possible accounts for their data. One is that participants may have shifted attention to peripheral natural images, perhaps more efficiently as a result of the training in their methodology. In short, it remains possible that efficient shift of attention to peripheral natural images masked the contribution of color. Another account is that, because direct response to natural images generates the sufficient activation of scene gist, it is not influenced by the color information. Previous studies (Delorme et al., 2000; Fei-Fei et al., 2005; Rousselet et al., 2005) reported no contribution of color information to scene recognition and adopted the direct response (e.g., a go vs. no-go task) to natural images. Although Goffaux et al. (2005) reported the contribution of color with direct response to natural images, scene gist might not be activated sufficiently because of the complexity of their four-alternative forced choice (4AFC) task. Indeed, Rousselet et al. partly reported the effect of color information with their 4AFC task. In addition, Castelhano and Henderson's (2008) study of scene recognition suggests that sufficient activation of scene gist masks the contribution of color. On the basis of this logic, we predict that color information contributes to the processing of natural scenes with (1) minimal attention to natural scenes or (2) indirect processing of ignored natural scenes, both providing insufficient activation of scene gist. Therefore, focusing on these points, we decided to examine whether color information would play an important role of natural scene categorization in an experimental design in which participants were not given massive training and did not respond directly to peripheral stimuli.

We used an NP paradigm (cf. Lavie \& Fox, 2000; see also Otsuka \& Kawaguchi, 2007) in which participants made similar responses while the amount of attentional resources allocated to the central stimuli was different in two attention-load conditions (low and high). Lavie and Fox found that NP was influenced by the attentional resources allocated to the prime display. NP was eliminated when the set size for visual search was larger than about four items, which apparently exhausted the resources for searching the central stimuli. Thus, we used set sizes of one and five for the low and high attention-load conditions, respectively. This procedure allows us to know whether the peripheral stimulus, which participants do not attend to, is processed. VanRullen, Reddy, and Koch (2004) proposed that objects in natural scenes do not pop out in a visual search task, whereas, with a dual task, they can be discriminated from distractors in the near absence of attention. Thus, in this study, we expected to find an influence of attention load with natural scenes and to examine whether object categorization in natural scenes is performed with minimal attention or indirect processing for natural scenes. If color information plays an important role in natural scene categorization with minimal attention, then performance of visual search in the prime dis- play should be higher in the color condition than in the grayscale condition when the set size is five and the NP effect should not be observed in the high attention-load condition with grayscale scenes. If color contributes to scene categorization without direct processing of scenes, then we should not observe an effect of color in visual search of the prime display but, instead, should observe an NP effect in the probe display of color scenes.

\section{METHOD}

\section{Participants}

Twelve Japanese undergraduate students at Nagoya University ( 2 men, 10 women; age range $=18-20$ years) participated for course credit. All were right-handed and reported normal or corrected-tonormal visual acuity and normal color vision on the basis of the Ishihara test of color blindness. All participants signed informed consent forms.

\section{Apparatus and Stimuli}

This experiment was run on a Macintosh computer (Apple, M9747J/A) controlled by the time schedule of PsyScope X (Cohen, MacWhinney, Flatt, \& Provost, 1993). Visual stimuli were presented on a color monitor (Mitsubishi, RDF221H; refresh rate $=100 \mathrm{~Hz}$; image resolution $=1,024 \times 768$ pixels). Participants viewed the computer screen from a distance of about $80 \mathrm{~cm}$. Participants responded using a button box, and their responses were measured using the response unit of PsyScope (ioLab Systems).

A total of 2,304 color images were selected from commercially available CD-ROMs of photographs (see Figure 1). The categories of target objects were animal (336 images; e.g., fish, mammals, birds, insects, and reptiles), vehicle (336 images; e.g., electric trains, airplanes, ships, and cars), and food (336 images; e.g., fruits, grains, and vegetables). Target images contained at least one target object. Searched nontargets in the high attention-load condition included such images as natural landscapes and city scenes that did not contain any object belonging to the three target categories (864 images). Distractor images on the probe display contained space, planet, and starlit skies (432 images) that were not associated with any response in this experiment. Participants had no knowledge about the size, position, or number of the targets in a single image, and these natural images were not presented more than once, except for a critical stimulus in the IR condition and a target in the attended repetition (AR) condition within a given trial. None of the images had been seen by the participants. An image subtended a visual angle of about $2.9^{\circ}$ vertically and about $2.9^{\circ}$ horizontally. In the grayscale condition, color information was removed using Adobe Photoshop. The luminance values of all grayscale images were equated to the same values as those of corresponding color images (about $45 \mathrm{~cd} / \mathrm{m}^{2}$ ) measured with a luminance colormeter (Topcon, BM-7).

Each trial consisted of a prime display and a probe display. Priming effect from the irrelevant prime distractor was assessed on the subsequent probe display. Lavie and Fox (2000) found that NP was eliminated for a set size larger than four, for which participants could not allocate their attentional resources to peripheral stimuli because they exhausted resources for the searched stimuli. Then we used set sizes of one and five for the low and high attention-load conditions, respectively. In the low attention-load condition (Figure 2A), one target image and one distractor image appeared, and none of the searched nontargets was presented in the prime display. In the high attention-load condition (see Figure 2B), one target image was accompanied by four nontarget images. In both conditions, a target image was presented at one of five equally probable positions. On the prime display, all the distractors were incompatible with the response required to the current target, and all appeared randomly, equally often, and equally distant above or below the horizontal axis of the screen. The eccentricity between each searched image was 
Animal
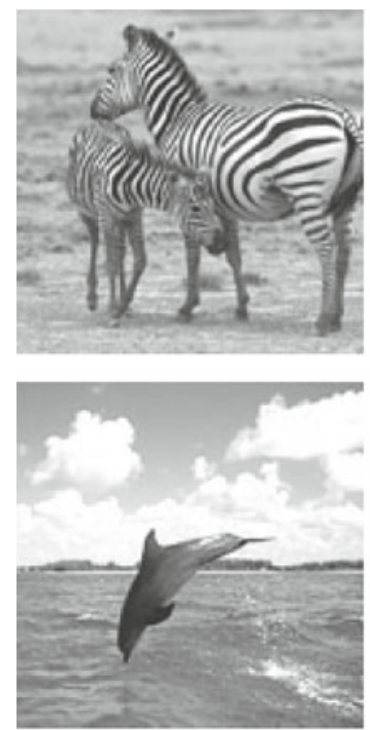

Vehicle
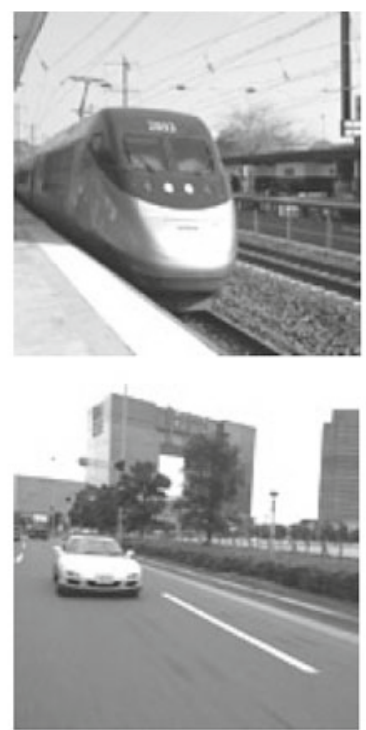

Food
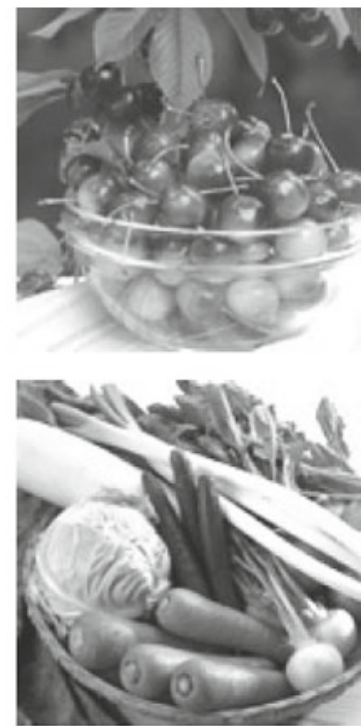

Nontargets
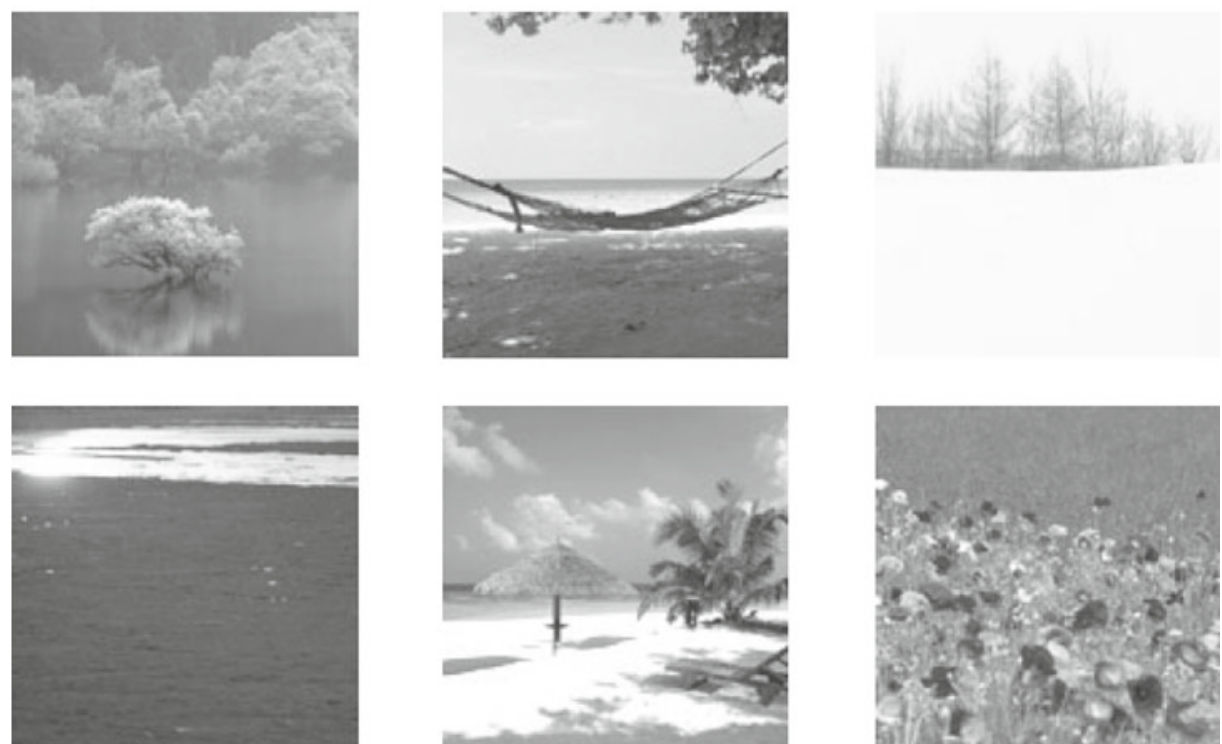

Figure 1. Examples of target pictures (animal, vehicle, and food) and nontarget pictures.

on average $3.2^{\circ}$, so that the eccentricity was about $6.4^{\circ}$ between the image presented at the center and at either end of the images and was about $12.8^{\circ}$ between both ends of the images. Also, the eccentricity between the ignored distractor image presented above or below the horizontal axis of the screen and the central image was about $4.3^{\circ}$, so that the eccentricity between the ignored distractor image and either of the images next to the central image was about $5.2^{\circ}$ and the eccentricity between the ignored distractor image and either end of the images was about $7.7^{\circ}$. Distractor images presented in the prime display changed with the NP conditions. In both the low and high attention-load conditions, a target image on the probe display appeared at one of two central locations to the immediate left or right of fixation, with one distractor that had no associated response in this experiment. The distractors were never presented in the same position between the prime and probe displays.

There were three priming conditions (see Figure 2). In all the displays, participants were required to respond to a target object in an image (animal, vehicle, food) as rapidly and accurately as possible, ignoring the image presented randomly above or below the horizontal axis of the screen. In the AR condition, the probe display contained a target image that was identical to the target image in the prime display. The distractor images always differed between the prime and probe displays (e.g., a target food image with a distractor vehicle image, followed by a target food image with a distractor space image). In the $\mathrm{C}$ condition, the probe display contained target and distractor images that were different from those in the prime display (e.g., a target 


\section{A. Low Attention Load}

IR
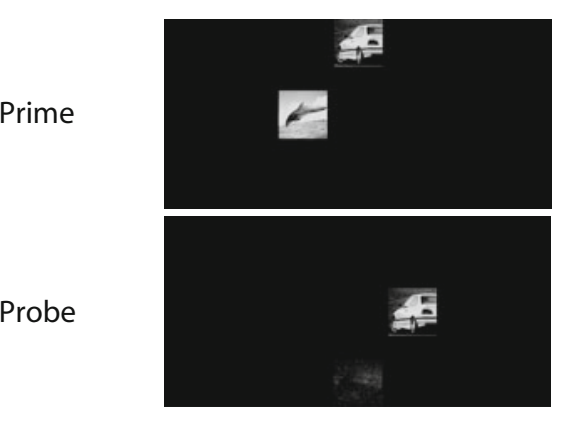

Probe
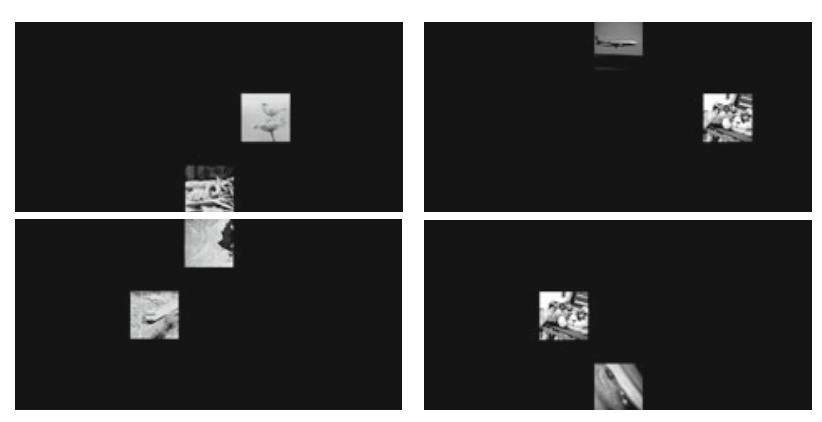

\section{B. High Attention Load}

IR

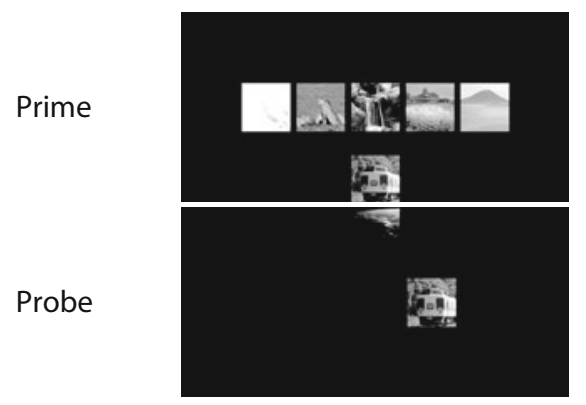

C

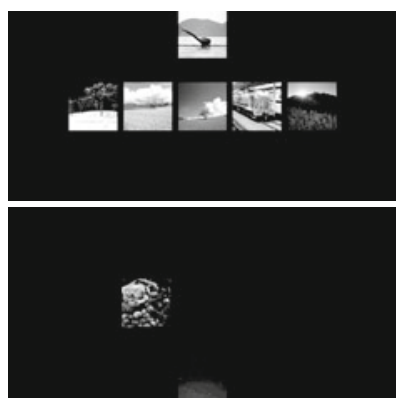

AR

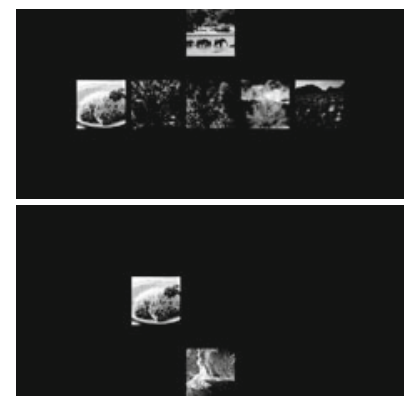

Figure 2. Sample displays from the low (A) and high (B) attention-load conditions. Participants were required to respond to a target object in an image (animal, vehicle, food) as rapidly and accurately as possible, ignoring the image presented randomly above or below the horizontal axis of the screen. IR, ignored repetition; C, control; AR, attended repetition.

vehicle image with a distractor animal image followed by a target food image with a distractor starlit skies image). In the IR condition, a target on the probe display had the same identity as the distractor in the previous prime display (e.g., a target animal image with a distractor vehicle image followed by a target vehicle image with a distractor planet image). We measured NP by subtracting the observed data in the IR condition from the corresponding data in the $\mathrm{C}$ condition. We made 12 lists of natural images, because of the counterbalance across participants (e.g., some images that were presented for 1 participant in the color, IR, and high attention-load condition were presented for another participant in the grayscale, $\mathrm{C}$, and low attention-load condition). In addition, we selected 576 images ( 84 for animal, 84 for vehicle, 84 for food, 216 for searched nontargets, and 108 for distractors on the probe display) for the practice trials that were not presented in the experimental trials.

In line with the procedure of Lavie and Fox (2000), we included a condition of target repetition across prime and probe displays (AR condition), because the previous studies of NP have shown that NP is generally larger when a proportion of trials included repetition of the target item across the prime and probe displays (e.g., Kane, May, Hasher, Rahhal, \& Stoltzfus, 1997).

\section{Design and Procedure}

The manipulated variables were type of color (color and grayscale), attention load (low and high), and relationship between the prime and probe displays (IR, C, and AR). All variables were withinsubjects factors.

The experiment was divided into 16 blocks, and each block comprised 27 trials. The relationship between the prime and probe displays was randomly presented within blocks (IR $=9$ trials, $\mathrm{C}=9$ trials, $\mathrm{AR}=9$ trials). Within 1 block, three target stimuli (animal, vehicle, food) were equally presented. Each participant received 432 trials (108 trials of color and high load, 108 trials of color and low load, 108 trials of grayscale and high load, and 108 trials of grayscale and low load). The order of type of color and attention load was counterbalanced across participants. Participants were required to respond to a target stimulus by pressing the key with their dominant hand (e.g., for the target animal image with their index finger, the target vehicle image with their second finger, and the target food image with their third finger), and the arrangement of the keys was counterbalanced across participants (participants always responded with their dominant hand). Following the instructions for this experiment, participants had 27 practice trials before they performed the task of each experimental condition. The conditions of attention load and type of color were presented in separate blocks of trials.

The trial schedule of the experiment was as follows (see Figure 3). A fixation cross was presented at the center of the screen for $500 \mathrm{msec}$. This was immediately replaced by the prime display, which appeared for $300 \mathrm{msec}$. Using the button box, participants were required to respond to a target object in an image as rapidly and accurately as possible by pressing the key allocated to category type (animal, vehicle, or food). Following the response to the prime display, the screen was blank for $300 \mathrm{msec}$, and then the probe display was presented for $300 \mathrm{msec}$. The response for the probe display was the same as that for the prime display. Participants used the same response-key mapping for the prime and probe displays. After their response to the probe display, a 1,000-msec intertrial interval preceded the next trial. The most important instruction was for participants to ignore the image presented randomly above or below the horizontal axis of the screen. 


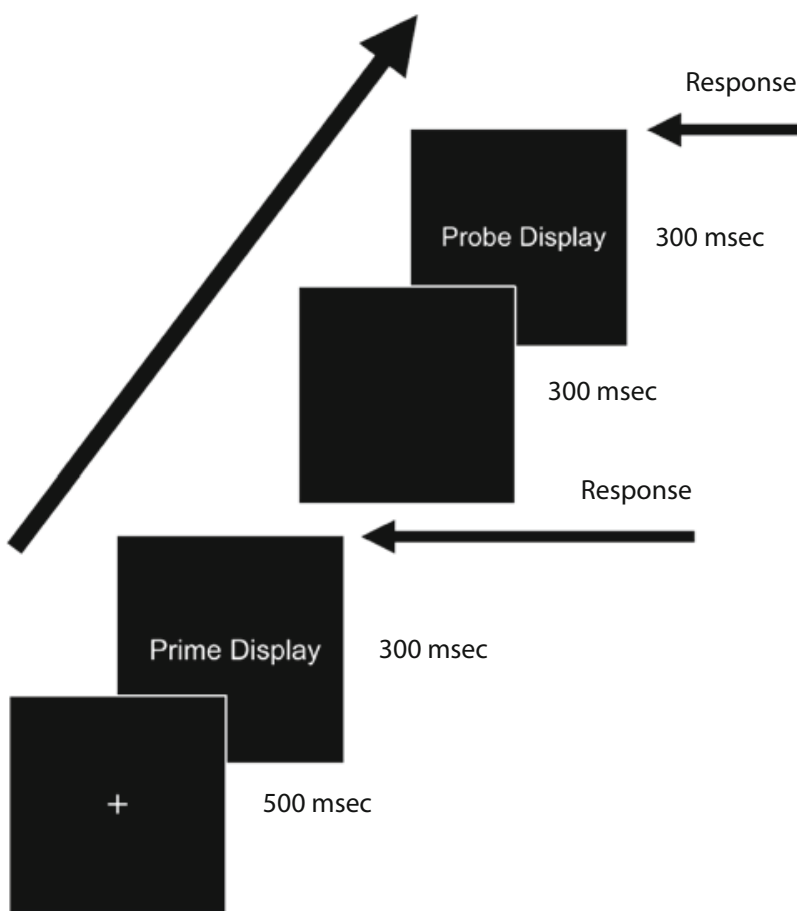

Figure 3. Trial schedule in this experiment.

\section{RESULTS}

The mean RTs and error rates are presented in Table 1 for the prime display and in Table 2 for the probe display as functions of the experimental conditions. Trials with errors and trials preceded by an error on the prime display were excluded from the probe RT analysis. Also, responses that deviated by at least 2 SDs from an individual participant's means were excluded. This trimming procedure was performed independently for each participant and for each condition, eliminating $4.0 \%$ of the responses in the prime display and $4.4 \%$ of the responses in the probe display.

We first analyzed the data of the prime display. A twoway within-subjects ANOVA was performed on the mean correct RTs and on the mean error rates, with the factors being type of color (color vs. grayscale) and attention load (low vs. high). The RT analysis showed a significant main effect of attention load $\left[F(1,11)=125.52, M S_{\mathrm{e}}=\right.$ $\left.1,083.31, p<.001, \eta_{\mathrm{p}}^{2}=.919\right]$ but no significant effect of type of color and no interaction between type of color and attention load $(F \mathrm{~s}<1)$. RTs in the high attention-load condition $(869 \mathrm{msec})$ were longer than those in the low attention-load condition $(763 \mathrm{msec})$. Also, the error data showed the significant effect of attention load $[F(1,11)=$ $\left.88.44, M S_{\mathrm{e}}=29.16, p<.001, \eta_{\mathrm{p}}^{2}=.889\right]$ but no significant effect of type of color and no interaction between type of color and attention load $\left[F(1,11)=1.92, M S_{\mathrm{e}}=\right.$ $16.43, p=.19, \eta_{\mathrm{p}}^{2}=.148$, and $F<1$, respectively]. The percentage of errors in the high attention-load condition $(20.8 \%)$ was higher than that in the low attention-load condition $(6.1 \%)$. These results indicate that the attention load was effectively manipulated, as we expected.
Next, we analyzed the data of the probe display. A threeway within-subjects ANOVA on the mean correct RTs, with the three factors being type of color (color vs. grayscale), attention load (low vs. high), and the critical relationship between the prime and probe displays (IR vs. C), indicated only a significant interaction between relationship and type of color $\left[F(1,11)=5.64, M S_{\mathrm{e}}=1,837.33\right.$, $\left.p<.05, \eta_{\mathrm{p}}^{2}=.339\right]$. An examination of the simple main effects revealed that the effect of displays was significant in the color condition, but not in the grayscale condition $\left[F(1,22)=8.36, M S_{\mathrm{e}}=1,309.57, p<.01, \eta_{\mathrm{p}}^{2}=.351\right.$, and $F(1,22)=1.18, M S_{\mathrm{e}}=1,309.57, p=.29, \eta_{\mathrm{p}}^{2}=.071$, respectively]. Thus, we observed a $-37-\mathrm{msec}$ NP effect in the low attention-load condition and a -24-msec NP effect in the high attention-load condition. A three-way withinsubjects ANOVA on the mean percentage of errors, with the three factors being type of color (color vs. grayscale), attention load (low vs. high), and the critical relationship between the prime and probe displays (IR vs. C), showed only a main effect of attention load $[F(1,11)=17.99$, $\left.M S_{\mathrm{e}}=8.65, p<.01, \eta_{\mathrm{p}}^{2}=.621\right]$. The rate of errors in the low attention-load condition (3.6\%) was higher than that in the high attention-load condition (1.9\%).

To test whether our results would depend on a particular type of display and category, we also conducted a fourway within-subjects ANOVA on the mean correct RTs and mean percentages of errors for the prime display, with type of color (color vs. grayscale), attention load (low vs. high), relationship between the prime and probe displays (IR, C, vs. AR), and category (animal, vehicle, vs. food) as factors. The results of RT data showed the significant interaction between attention load and category $\left[F(2,22)=3.81, M S_{\mathrm{e}}=\right.$ $\left.6,432.06, p<.05, \eta_{\mathrm{p}}^{2}=.257\right]$, but the effect of attention load was significant in all categories $[F(1,33)=65.11$, $M S_{\mathrm{e}}=7,603.50, p<.001, \eta_{\mathrm{p}}^{2}=.778 ; F(1,33)=29.04$, $M S_{\mathrm{e}}=7,603.50, p<.001, \eta_{\mathrm{p}}^{2}=.609 ;$ and $F(1,33)=$ $77.34, M S_{\mathrm{e}}=7,603.50, p<.001, \eta_{\mathrm{p}}^{2}=.806$, respectively]. We did not observe any interaction including attention load or relationship between the prime and probe displays. But the results of the error data showed that the threeway interaction including type of color, attention load, and category was significant $\left[F(2,22)=4.11, M S_{\mathrm{e}}=65.22\right.$, $\left.p<.05, \eta_{\mathrm{p}}^{2}=.272\right]$, which indicated that the effects of category were significant in the high attention-load condition, both with color and grayscale stimuli $[F(2,88)=$ $3.15, M S_{\mathrm{e}}=151.05, p<.05, \eta_{\mathrm{p}}^{2}=.339$, and $F(2,88)=$ $3.48, M S_{\mathrm{e}}=151.05, p<.05, \eta_{\mathrm{p}}^{2}=.423$, respectively]. Tukey's HSD test indicated that percentage of errors for food $(15.5 \%)$ was lower than that for animals $(22.2 \%)$ in the high attention-load condition with color stimuli, and percentage of errors for vehicles $(17.6 \%)$ was lower than that for food $(25.0 \%)$ in the high attention-load condition with grayscale stimuli (HSD $=6.03$ ). However, in addition, we observed the effect of attention load all in the experimental conditions including category. These results do not indicate that attention load in the prime display depended on a particular type of display and category. Next, we conducted a four-way within-subjects ANOVA on the mean correct RTs and mean percentages of errors 
Table 1

Mean Reaction Times (RTs, in Milliseconds), Standard Errors (SEs), and Percentage of Errors (PEs) in the Prime Display As a Function of Color of Images and Attention Load

Attention Load

\begin{tabular}{|c|c|c|c|c|c|c|c|c|}
\hline \multirow[b]{4}{*}{ Type of Color } & \\
\hline & \multicolumn{4}{|c|}{ Low } & \multicolumn{4}{|c|}{ High } \\
\hline & \multicolumn{2}{|c|}{ RT } & \multicolumn{2}{|c|}{$\mathrm{PE}$} & \multicolumn{2}{|c|}{ RT } & \multicolumn{2}{|c|}{$\mathrm{PE}$} \\
\hline & $M$ & $\overline{S E}$ & $M$ & $\overline{S E}$ & $M$ & $\overline{S E}$ & $M$ & $\overline{S E}$ \\
\hline Color & 759 & 37 & 5.6 & 1.5 & 875 & 40 & 19.7 & 1.5 \\
\hline Grayscale & 767 & 33 & 6.6 & 1.0 & 863 & 41 & 21.8 & 1.7 \\
\hline
\end{tabular}

for the probe display, with type of color (color vs. grayscale), attention load (low vs. high), the critical relationship between the prime and probe displays (IR vs. C), and category (animal, vehicle, vs. food) as factors. The results of RT and percentage of errors data did not show any interaction including category. ${ }^{2}$ These results do not indicate that NP in the probe display depended on a particular type of category.

We also performed an analysis on the AR condition, running a three-way within-subjects ANOVA with the factors being type of color (color vs. grayscale), attention load (low vs. high), and relationship (AR vs. C). The RT data showed significant effects of attention load and relationship $\left[F(1,11)=5.81, M S_{\mathrm{e}}=1,625.90, p<.05\right.$, $\eta_{\mathrm{p}}^{2}=.346$, and $F(1,11)=115.54, M S_{\mathrm{e}}=8,367.44, p<$ $.001, \eta_{\mathrm{p}}^{2}=.913$, respectively]. Also, the data for percentage of errors showed a significant effect of relationship $\left[F(1,11)=30.41, M S_{\mathrm{e}}=9.20, p<.001, \eta_{\mathrm{p}}^{2}=.734\right]$. These results can be interpreted as being typical positive repetition priming effects, which is consistent with the findings of Lavie and Fox (2000), in which a positive priming effect was shown irrespective of attention load, although they showed greater positive priming from AR targets with higher load in their Experiment 1.

\section{DISCUSSION}

The present study examined whether color and grayscale natural scenes presented peripherally and ignored would be categorized using an NP paradigm (cf. Lavie \& Fox, 2000); we focused on (1) attentional resources allocated to scenes and (2) direct versus indirect processing of scenes. The main finding of the present study is that NP from the ignored images was observed in color natural scenes irrespective of attention load, which was consistent with the findings of a previous study (Otsuka \& Kawaguchi, 2007), whereas NP was not observed in grayscale natural scenes. In addition, a contribution of color information was not found in central visual search of the prime display. These results suggest that, independent of attention load, the effect of color information is found with the indirect processing of peripheral natural scenes. In addition, these results can suggest that the contribution of color information depends not on scene alone (e.g., Oliva \& Schyns, 2000) but also on experimental-condition-dependent factors (i.e., direct vs. indirect processing).

Table 3 shows the previous studies of natural scene categorization addressing color influence. On the whole, previous studies in which exposure duration of natural images was very short (about $30 \mathrm{msec}$ ) and participants preformed a go versus no-go task and directly responded to target images did not report the contribution of color information (e.g., Delorme et al., 2000; Fei-Fei et al., 2005; Rousselet et al., 2005). On the other hand, previous studies in which the exposure duration of natural images was relatively long (about $100 \mathrm{msec}$ ) and the responses of participants to target images were not directly different from those in a go versus no-go task (e.g., contextual bias paradigm, recognition memory, and naming with 4AFC task) reported the contribution of color information (e.g., Castelhano \& Henderson, 2008; Goffaux et al., 2005; Oliva \& Schyns, 2000, excepting the shorter exposure durations of Spence et al., 2006). With regard to the exposure duration of a natural image, if the longer duration (about $100 \mathrm{msec}$ ) is sufficient to generate the color effect, we should find an effect of color in the central visual task for the prime display. However, we did not find one, so it may not be possible that only the factor of exposure duration interacts with the contribution of color information. Our findings and evidence from these previous studies suggest that it is possible that one important factor for the contribution of color information in natural scene recognition is direct versus indirect processing of natural images. More recently, Castelhano and Henderson have suggested that the findings that there is no contribution of color information with normal scenes, as compared with blurred scenes, may be attributed to sufficient processing of scene gist and that this masks the effect of color. In the prime display of our experiment, it might be that direct processing of natural images allowed sufficient activation of scene gist,

Table 2

Mean Reaction Times (RTs, in Milliseconds), Standard Errors (SEs), and Percentage of Errors (PEs) in the Probe Display As a Function of Each Experimental Condition

\begin{tabular}{|c|c|c|c|c|c|c|c|c|c|c|c|c|c|c|c|c|}
\hline \multirow[b]{4}{*}{ Condition } & \multicolumn{8}{|c|}{ Color } & \multicolumn{8}{|c|}{ Grayscale } \\
\hline & \multicolumn{4}{|c|}{ Low } & \multicolumn{4}{|c|}{ High } & \multicolumn{4}{|c|}{ Low } & \multicolumn{4}{|c|}{ High } \\
\hline & \multicolumn{2}{|c|}{ RT } & \multicolumn{2}{|c|}{$\mathrm{PE}$} & \multicolumn{2}{|c|}{ RT } & \multicolumn{2}{|c|}{$\mathrm{PE}$} & \multicolumn{2}{|c|}{ RT } & \multicolumn{2}{|c|}{ PE } & \multicolumn{2}{|c|}{ RT } & \multicolumn{2}{|c|}{$\mathrm{PE}$} \\
\hline & $M$ & $S E$ & $M$ & $S E$ & $M$ & $S E$ & $M$ & $S E$ & $M$ & $S E$ & $M$ & $S E$ & $M$ & $S E$ & $M$ & $S E$ \\
\hline Ignored repetition & 731 & 31 & 4.9 & 1.9 & 716 & 28 & 1.9 & 0.6 & 702 & 24 & 5.8 & 1.2 & 709 & 25 & 2.5 & 0.9 \\
\hline Control & 695 & 30 & 4.4 & 0.8 & 692 & 24 & 2.5 & 0.9 & 721 & 31 & 5.6 & 1.5 & 713 & 35 & 3.5 & 1.0 \\
\hline Attended repetition & 484 & 19 & 0.7 & 0.4 & 531 & 35 & 0.7 & 0.4 & 480 & 21 & 0.5 & 0.3 & 523 & 25 & 0.5 & 0.3 \\
\hline Control - ignored repetition & $-37^{*}$ & & -0.5 & & $-24^{*}$ & & 0.7 & & 19 & & -0.2 & & 4 & & 1.0 & \\
\hline
\end{tabular}

${ }^{*} p<.01$. 
and the contribution of color information was masked, whereas indirect processing of peripheral natural images did not generate sufficient activation of scene gist, so that participants ignored and did not respond to peripheral natural images directly. This idea can explain the difference between no NP in the low attention-load condition of grayscale images and no effect of color in the prime display. On the basis of this idea, we can consistently account for the data of Fei-Fei et al. and for our findings for prime and probe displays. Probably, relative amount of attentional resources allocated to processing of natural scenes might not be critical for sufficient activation of scene gist. In summary, our research and previous studies indicate that direct versus indirect processing of natural images determines the contribution of color information in natural scene categorization, implying that, when activation of scene gist is not sufficient on the basis of the task and scene themselves (e.g., Castelhano \& Henderson, 2008; Goffaux et al., 2005; Spence et al., 2006) - in addition to the attention load, exposure duration, and massive training - color information may contribute to natural scene categorization.

In terms of neuroimaging studies, the processing of blurred natural images may be related to the cognitive and cortical mechanism proposed by Bar $(2003,2004)$. Bar proposed a hypothetical model for the rapid visual processing of natural scene categorization. According to his model, the visual system rapidly detects low-frequency information ("coarse information") in natural scenes. This blurred information is believed to be projected from $\mathrm{V} 2$ and V4 to the prefrontal cortex (PFC), where possible candidates for the target objects are selected. This blurred information is also projected to the parahippocampal cortex (PHC), where the visual system selects the most likely context for the natural images. A few candidates for the target objects and the context frame are projected to the inferior temporal cortex (ITC), where the target object in the natural scene is identified. Such a series of processes has been hypothesized as underlying the recognition of natural scenes. According to this hypothesis, coarse information about a target object contains color information, and this information can be processed irrespective of attentional resources because the coarse information is projected from V2 and V4, which are concerned with the processing of color information (e.g., Kiper, Fenstemaker, \& Gegenfurtner, 1997; Zeki, 1973, 1983), to the ventral PFC. With colored natural images, participants might extract coarse information from the peripheral natural scene on the prime display, even if they do not directly and consciously respond to peripheral scenes. This coarse information will be projected to the PFC and PHC, where a few candidates for the target objects and the context frame will be selected, which then will be projected to the ITC. This process will generate the categorization of the natural scene on the prime display without direct response. Hence, this information will affect the participants' subsequent responses to the probe display, including the occurrence of the NP effect (Otsuka \& Kawaguchi, 2007). In this respect, our study may support the previous findings that the influence of color interacts with the influence of structure information (Castelhano \& Henderson, 2008; Oliva \& Schyns, 2000).

We employed an NP paradigm in our present study. In general, NP is explained by two theories. One is based on inhibition (e.g., Houghton \& Tipper, 1994; Neill, 1977; Tipper, 1985; Tipper \& Cranston, 1985; Tipper \& Driver, 1988; Yee, 1991). According to the inhibitory explanation, when people have to selectively attend to a stimulus or stimulus dimension, their attention mechanisms emphasize the target element and simultaneously suppress the representations of task-irrelevant information. This inhibitory view addresses the benefit of highlighting the

Table 3

Previous Studies Addressing the Relationship Between Natural Scene Categorization and the Contribution of Color Information

\begin{tabular}{|c|c|c|c|c|}
\hline Article & $\begin{array}{l}\text { Exposure } \\
\text { Duration }\end{array}$ & $\begin{array}{c}\text { Task } \\
\text { (or Procedure) }\end{array}$ & Practice & Results \\
\hline Castelhano \& Henderson (2008) & $\begin{array}{l}20,50,80,100 \\
\text { and } 250 \mathrm{msec}\end{array}$ & $\begin{array}{l}\text { Contextual bias } \\
\text { paradigm }\end{array}$ & Unclear & $\begin{array}{l}\text { Color effect of blurred images at durations of } \\
80,100 \text {, and } 250 \mathrm{msec}^{\mathrm{a}}\end{array}$ \\
\hline Goffaux et al. (2005) & $100 \mathrm{msec}$ & Go/no-go task & Unclear & Color effect \\
\hline Oliva \& Schyns (2000) & $120 \mathrm{msec}$ & $\begin{array}{l}\text { Naming with } \\
8 \text { AFC task }\end{array}$ & 8 trials $^{\mathrm{b}}$ & $\begin{array}{l}\text { Color effect in the diagnostic condition (RT) } \\
\text { and in the diagnostic condition of coarse } \\
\text { stimuli (accuracy)c }\end{array}$ \\
\hline $\begin{array}{l}\text { Spence, Wong, Rusan, } \\
\text { \& Rastegar (2006) }\end{array}$ & $\begin{array}{l}20,32,48,64 \\
96,128,300 \\
\text { and } 2,000 \mathrm{msec}\end{array}$ & $\begin{array}{l}\text { Recognition } \\
\text { task }\end{array}$ & Unclear & Color effect (at all exposure durations) \\
\hline $\begin{array}{l}\text { Delorme, Richard, \& Fabre-Thorpe } \\
\text { (2000) }\end{array}$ & $32 \mathrm{msec}$ & Go/no-go task & $\_\mathrm{d}$ & Noncolor effect \\
\hline $\begin{array}{l}\text { Fei-Fei, VanRullen, Koch, \& Perona } \\
\text { (2005) }\end{array}$ & $\begin{array}{l}27 \text { msec with } \\
\text { masking }\end{array}$ & $\begin{array}{l}\text { Go/no-go task } \\
\text { with dual task }\end{array}$ & More than $10 \mathrm{~h}$ & Noncolor effect \\
\hline $\begin{array}{l}\text { Rousselet, Joubert, \& Fabre-Thorpe } \\
\text { (2005) }\end{array}$ & $26 \mathrm{msec}$ & Go/no-go task & 48 trials & Noncolor effect \\
\hline
\end{tabular}

aCastelhano and Henderson (2008) used the blurred scenes created by using a low-pass filter at 1 cpd of visual angle. bIn Experiment 1, practice trials in other experiments were not described. coliva and Schyns (2000) used low-passed colored scenes starting with a cutoff of 0.5 cpd climbing progressively to $8 \mathrm{cpd}$ of visual angle. dTen images, with more images added gradually over a period of several weeks until monkeys were performing the categorization task well. 
target on the prime trial, at the expense of making it more difficult to use the suppressed representations shortly thereafter on the probe trial.

Another explanation of NP is the episodic trace theory, which emphasizes memory retrieval and does not assume an inhibitory mechanism (e.g., Neill \& Valdes, 1992; Neill, Valdes, Terry, \& Gorfein, 1992). Interference is considered to result from the response conflict between the "do not respond" tag attached to the ignored prime item and the "respond" tag attached to the target probe item (the same item). When the target probe is encountered, retrieval of recent relevant information occurs, but the item most likely to be retrieved (the ignored prime item) carries a tag that disrupts responding to the same item, now the target probe.

We consider that both the inhibitory theory and the episodic trace theory can adequately explain the NP in our procedure for color scenes in the low and high attentionload conditions. According to the inhibitory theory, the ignored natural images were processed with minimal or less attention and were inhibited. Because a target on the probe display had the same identity as the distractor in the previous prime display, the NP could also be explained using the episodic trace theory. For the same reason, the NP effects observed in the low attention-load condition could be explained by either the inhibitory or the episodic trace theory. On the other hand, with respect to the performance in the grayscale condition, some might argue that NP was not observed in the low attention-load condition so that participants did not have any attentional resources to allocate to peripheral grayscale images. However, the results of RTs and accuracy data for the prime display did not show the interaction between type of color and attention load, implying that participants were able to have their resources allocated to peripheral grayscale scenes to the same extent as for peripheral color scenes.

In conclusion, human visual processes need the color information of scenes to process targets in scenes to which they cannot respond directly, irrespective of their attentional resources. The effect of color information would be important for the reason that images are similar to the scenes of daily vision, and so this information should play an important role in indirect processing of targets in scenes.

\section{AUTHOR NOTE}

We thank Jeremy M. Wolfe, Shaun Vecera, Dale Dagenbach, and three anonymous reviewers for their insightful comments on an earlier draft of the manuscript. We also thank Hayaki Banno (Kyoto University) and Hiroshi Ro (Nagoya City University) for good advice about evaluating contrast. Address correspondence concerning this article to S. Otsuka or J. Kawaguchi, Department of Psychology, Graduate School of Environmental Studies, Nagoya University, Furo-cho, Chikusa-ku, Nagoya, 464-8601, Japan (e-mail: otsuka.sachio@f.mbox.nagoya-u.ac.jp or kawaguchijun@nagoya-u.jp).

\section{REFERENCES}

Bacon-Macé, N., Macé, M. J.-M., Fabre-Thorpe, M., \& Thorpe, S. J. (2005). The time course of visual processing: Backward masking and natural scene categorization. Vision Research, 45, 1459-1469. doi:10.1016/j.visres.2005.01.004

BAR, M. (2003). A cortical mechanism for triggering top-down facilita- tion in visual object recognition. Journal of Cognitive Neuroscience, 15, 600-609. doi:10.1162/089892903321662976

BAR, M. (2004). Visual objects in context. Nature Reviews Neuroscience, 5, 617-629. doi:10.1038/nrn1476

Castelhano, M. S., \& Henderson, J. M. (2008). The influence of color on the perception of scene gist. Journal of Experimental Psychology: Human Perception \& Performance, 34, 660-675. doi:10.1037/0096 $-1523.34 .3 .660$

Cohen, J., MacWhinney, B., Flatt, M., \& Provost, J. (1993). PsyScope: An interactive graphic system for designing and controlling experiments in the psychology laboratory using Macintosh computers. Behavior Research Methods, Instruments, \& Computers, 25, 257-271.

Delorme, A., Richard, G., \& Fabre-Thorpe, M. (2000). Ultrarapid categorisation of natural scenes does not rely on colour cues: A study in monkeys and humans. Vision Research, 40, 2187-2200. doi:10.1016/S0042-6989(00)00083-3

Evans, K. K., \& Treisman, A. (2005). Perception of objects in natural scenes: Is it really attention-free? Journal of Experimental Psychology: Human Perception \& Performance, 31, 1476-1492. doi:10.1037/0096 $-1523.31 .6 .1476$

Fei-Fei, L., VanRullen, R., Koch, C., \& Perona, P. (2005). Why does natural scene categorization require little attention? Exploring attentional requirements for natural and synthetic stimuli. Visual Cognition, 12, 893-924. doi:10.1080/13506280444000571

Goffaux, V., Jacques, C., Mouraux, A., Oliva, A., Schyns, P. G., \& Rossion, B. (2005). Diagnostic colours contribute to the early stages of scene categorization: Behavioural and neurophysiological evidence. Visual Cognition, 12, 878-892. doi:10.1080/13506280444000562

Houghton, G., \& TipPER, S. P. (1994). A model of inhibitory mechanisms in selective attention. In D. Dagenbach \& T. H. Carr (Eds.), Inhibitory processes in attention, memory, and language (pp. 53-112). San Diego: Academic Press.

Kane, M. J., May, C. P., Hasher, L., Rahhal, T., \& Stoltzfus, E. R. (1997). Dual mechanisms of negative priming. Journal of Experimental Psychology: Human Perception \& Performance, 23, 632-650. doi:10.1037/0096-1523.23.3.632

Kiper, D. C., Fenstemaker, S. B., \& Gegenfurtner, K. R. (1997). Chromatic properties of neurons in macaque area V2. Visual Neuroscience, 14, 1061-1072.

Lavie, N., \& Fox, E. (2000). The role of perceptual load in negative priming. Journal of Experimental Psychology: Human Perception \& Performance, 26, 1038-1052. doi:10.1037/0096-1523.26.3.1038

Li, F. F., VanRullen, R., Кoch, C., \& Perona, P. (2002). Rapid natural scene categorization in the near absence of attention. Proceedings of the National Academy of Sciences, 99, 9596-9601. doi:10.1073 pnas.092277599

NEILL, W. T. (1977). Inhibitory and facilitatory processes in selective attention. Journal of Experimental Psychology: Human Perception \& Performance, 3, 444-450. doi:10.1037/0096-1523.3.3.444

NeILL, W. T., \& Valdes, L. A. (1992). Persistence of negative priming: Steady state or decay? Journal of Experimental Psychology: Learning, Memory, \& Cognition, 18, 565-576. doi:10.1037/0278 $-7393.18 .3 .565$

Neill, W. T., Valdes, L. A., Terry, K. M., \& Gorfein, D. S. (1992). Persistence of negative priming: II. Evidence for episodic trace retrieval. Journal of Experimental Psychology: Learning, Memory, \& Cognition, 18, 993-1000. doi:10.1037/0278-7393.18.5.993

Oliva, A., \& Schyns, P. G. (2000). Diagnostic colors mediate scene recognition. Cognitive Psychology, 41, 176-210. doi:10.1006/ cogp.1999.0728

OtsuKa, S., \& KaWAGUChI, J. (2007). Natural scene categorization with minimal attention: Evidence from negative priming. Perception \& Psychophysics, 69, 1126-1139.

Rousselet, G. A., Joubert, O. R., \& Fabre-Thorpe, M. (2005). How long to get the "gist" of real-world natural scenes? Visual Cognition, 12, 852-877. doi: $10.1080 / 13506280444000553$

Spence, I., Wong, P., Rusan, M., \& Rastegar, N. (2006). How color enhances visual memory for natural scenes. Psychological Science, 17, 1-6. doi:10.1111/j.1467-9280.2005.01656.x

Thorpe, S., Fize, D., \& Marlot, C. (1996). Speed of processing in the human visual system. Nature, 381, 520-522. doi:10.1038/381520a0 
TIPPER, S. P. (1985). The negative priming effect: Inhibitory priming by ignored objects. Quarterly Journal of Experimental Psychology, 37A, 571-590.

TipPer, S. P., \& Cranston, M. (1985). Selective attention and priming: Inhibitory and facilitatory effects of ignored primes. Quarterly Journal of Experimental Psychology, 37A, 591-611.

TipPER, S. P., \& Driver, J. (1988). Negative priming between pictures and words in a selective attention task: Evidence for semantic processing of ignored stimuli. Memory \& Cognition, 16, 64-70.

VAnRullen, R., \& Koch, C. (2003). Visual selective behavior can be triggered by a feed-forward process. Journal of Cognitive Neuroscience, 15, 209-217. doi:10.1162/089892903321208141

VAnRullen, R., Reddy, L., \& Koch, C. (2004). Visual search and dual tasks reveal two distinct attentional resources. Journal of Cognitive Neuroscience, 16, 4-14. doi:10.1162/089892904322755502

VAnRullen, R., \& Thorpe, S. J. (2001a). Is it a bird? Is it a plane? Ultra-rapid visual categorisation of natural and artifactual objects. Perception, 30, 655-668. doi:10.1068/p3029

VAnRullen, R., \& Thorpe, S. J. (2001b). The time course of visual processing: From early perception to decision-making. Journal of Cognitive Neuroscience, 13, 454-461. doi:10.1162/08989290152001880

YeE, P. L. (1991). Semantic inhibition of ignored words during a figure classification task. Quarterly Journal of Experimental Psychology, 43A, 127-153.

ZeKI, S. M. (1973). Colour coding in rhesus monkey prestriate cortex. Brain Research, 53, 422-427. doi:10.1016/0006-8993(73)90227-8

ZEKI, S. [M.] (1983). The distribution of wavelength and orientation selective cells in different areas of monkey visual cortex. Proceedings of the Royal Society B, 217, 449-470.

\section{NOTES}

1. Oliva and Schyns (2000) used low-passed colored scenes starting with a cutoff of $0.5 \mathrm{cpd}$ climbing progressively to $8 \mathrm{cpd}$ of visual angle, to examine the hypothesis that the addition of colors to luminance cues improves the efficiency of scene categorization.
2. We calculated the RMS contrast of all target images. With animal targets, the mean RMS contrast of color images was 49.5 and that of grayscale images was 43.2. With vehicle targets, the mean RMS contrast of color images was 60.7, and that of grayscale images was 60.5. With food targets, the mean RMS contrast of color images was 60.2 , and that of grayscale images was 59.0. If higher contrast images result in NP, we should observe significant NP in the vehicle and food targets compared with animal targets, irrespective of color condition. Alternatively, we should observe an NP effect only in the animal targets, because color images had higher contrast than grayscale images did in the animal condition. However, we did not observe the above results. We conducted a four-way ANOVA on the mean correct RTs of probe display, with type of color, attention load, the critical relationship between the prime and probe displays, and category as factors, and we did not observe any interaction including category. So the difference of contrast, but not that of color, is difficult to explain in our results.

3 . We calculated the mean RMS contrast of all target images (animal targets, color images $=49.5$, grayscale images $=43.2$; vehicle targets, color images $=60.7$, grayscale images $=60.5$; food targets, color images $=60.2$, grayscale images $=59.0$ ). If higher contrast images result in NP, we should observe significant NP in the vehicle and food targets, as compared with animal targets, irrespective of color condition. Alternatively, we should observe an NP effect only in the animal targets, because color images had higher contrast than grayscale images did in the animal condition. However, we did not observe such results. We conducted a four-way ANOVA on the mean correct RTs of probe display, with type of color, attention load, the critical relationship between the prime and probe displays, and category as factors, and we did not observe any interaction including category. So the difference of contrast, but not that of color, is difficult to explain with respect to our results.

(Manuscript received December 12, 2008; revision accepted for publication April 25, 2009.) 